\title{
Fabrication of multi-level 3-dimension microstructures by phase inversion process
}

\section{Y. Song*}

One process based on phase inversion of fillers in microstructures for the fabrication of multi-level three-dimensional (3-D) microstructures is described using SU-8, a kind of epoxy photoresist, as the model constructing materials. This process is depicted by use of the routine photolithography technique to construct the top layer of 3-D microstructures on the bottom layer of 3-D microstructures layer by layer. This process makes it possible to fabricate multi-level 3-D microstructures with connectors at desired locations, and to seal long span microstructures (e.g. very shallow channels with depth less than $50 \mu \mathrm{m}$ and width more than $300 \mu \mathrm{m}$ ) without blockage. In addition, this process can provide a sealing layer by the solidification of a liquid polymer layer, which can be as strong as the bulk constructing materials for microstructures due to a complete contact and cross-linking between the sealing layer and the patterned layers. The hydrodynamic testing indicates that this kind of sealing and interconnection can endure a static pressure of more than 10 MPa overnight and a hydrodynamic pressure drop of about 5.3 MPa for more than 8 hours by pumping the tetrahydrofuran solution through a $60 \mu \mathrm{m}$ wide micro-channels.

Keywords: Microstructures; Multi-level; Fabrication; Photolithography; Phase inversion; Sealing

Citation: Y. Song, "Fabrication of multi-level 3-dimension microstructures by phase inversion process", Nano-Micro Lett. 2, 95-100 (2010). doi: 10.5101/nml.v2i2.p95-100

Micro and nano fluidic devices are useful not only because they allow manipulation with fast response times, handle small fluid volumes, sense and control flows and pattern substrates on small lengths scale, but also promise selectively address the cellular cell $[1,2]$. As a result of the uniform reactor conditions in mass and heat transfer similar as in microbial cells obtained, a high degree of reaction control is observed [3,4]. Modern developments in the design and utilization of micro fluidic devices for fluid transport have found many applications, ranging from the life science industries for pharmaceuticals and biomedicine (drug discovery, drug delivery and detection, diagnostic devices) [4,5] to industrial applications of combinatorial synthesis (such as, stereoselective synthesis [3], nanoparticle synthesis [6-8], rapid in-situ chemical analyses and high throughput screening $[9,10])$. Hence, there is an urgent need for developing rapid and economically viable prototyping processes for manufacturing micro fluidic devices with suitable materials compatible with the application environment $[11,12]$.

In order to realize lab-on-a-chip in practical sense, micro-temperature controller (heat-exchanger and T-sensor), micro pressure controller, micro flow rate controller, micro separator, micro detector and other micro devices are necessarily integrated in one chip, most probably realized in multi-level 3-D microstructures with connectors at any locations. Building microstructures in 3-D still presents significant challenges when working with the inherently planar geometries that are accessible through projection photolithography [11-15]. Alternatives to photolithography for fabrication schemes that "write" patterns serially in metals and polymers either carve in a wise manner from a solid object or cause localized deposition of material in a series manner $[15,16]$. However, these methods are often limited in the connectivity and dimensionalities of structures they can 
generate. The idea by transferring 2-D patterns to 3-D microstructures through pseudo-3-D patterns in a cylindrically symmetrical substrate remains a challenge to form multi-level 3-D microstructures with connectors and lose the convenience to build complicated microstructures with more than one layer of 3-D structures on the normal chip [16].

Compared with silicon, glass or stainless steel based micro fluidic devices, polymer based micro devices are more promising as microchips because of the availability of different types of polymers, low cost and ease of fabrication using LIGA, embossing, casting, injection molding and imprinting process $[17,18]$. Careful selection of polymers and fabrication process can lead to commercially viable fabrication processes and different application environment for micro fluidic devices [12]. SU-8, an epoxy-based negative photo resist, has been gaining much attention as a material of choice for the fabrication of microstructures and microchannels due to its superior chemical and mechanical properties besides its ease of fabrication using photolithography process $[12,16,18-20]$. However, when using SU-8 for the fabrication of multi-level 3-D microstructures, there are still some issues that need to be addressed. The multi-level 3-D microstructures fabricated with connectors at desired locations, a strong binding interface for complete sealing of these microstructures and the sealing for long span microchannels with a shallow depth $(<50 \mu \mathrm{m})$ without any blockage are still not trivial problems although many processes have been developed for these problems $[11,12,16]$. The developed process by thermal binding two pre-patterned solid SU-8 layers to form multi-level 3-D microstructures needs to overcome the micro gas-gaps between the two solid layers or operate under vacuum and then to form a complete inter-connecting between the two layers, which is usually not a trivial matter $[11,12,21]$. Furthermore, it is difficult to get connecting parts at some desired locations between the two layers or needs tedious alignment [11,21]. In addition, once the substrate is used to support the SU-8 layer, additional work is required to release the substrate from the SU-8 layer for the construction of other levels. Otherwise, it will lose the opportunity to construct additional SU-8 layer on the pre-formed SU-8 layers [11].

This report demonstrates a flexible process based on the phase inversion of fillers in the pre-patterned microstructures for the design, the fabrication and the sealing of multi-level 3-D microstructures. This process favors to get free-selected connectors between two contacting layers and the strong sealing of the long span microstructures with depth less than $50 \mu \mathrm{m}$ using the routine photolithography process.
Figure 1 shows the schematic diagram for the fabrication of multi-level 3-D microstructures with freely designed connectors. The first layer with embedded structures is formed according to our previous process (see Fig. 1(i)) [12]. Then a kind of filler (i.e. wax, polymers, etc.) will be heated above its melting point and filled into the pre-formed microstructures (see Fig. 1(ii)) by a syringe at a large opening orifice. The second layer of liquid photo resist (e.g. SU-8) is coated on the filled first level of microstructures (see Fig. 1(iii)) after the liquid filler changes to solid. Using the routine photolithography process described in reference 12, the second level of microstructures can be constructed in the second layer of photo resist on the first level of microstructures (see Fig. 1(iv)). The second level of microstructures is then filled again by the fillers like step 2 (see Fig. 1(v)). Then the third layer of the liquid photo resist (e.g. SU-8) will be spin-coated on the top of the filled 2 levels of microstructures (see Fig. 1(vi)). Similar as previous steps, the third layer can be constructed and refilled by the fillers (see Fig. 1(vii)). If necessary, hundreds of levels of microstructures can be fabricated layer by layer (see Fig. 1(viii)) just following the previous steps (ii-iv). When all the desired microstructures with necessary levels are constructed, the whole microstructures can be further sealed according to their applications by constructing the last layer of photo resist using the routine photolithography process (see Fig. 1, ix-x). After all the constructions are completed, the whole multi-level 3-D microstructures can be increased to a certain temperature (above the melting points of fillers) to liquefy the fillers. Then the liquid fillers can be pushed out of the whole microstructured chip by compressed air flow. If necessary, the sealed or opened microstructures can be washed by a certain solvent to dissolve the fillers on the walls of microstructures to make sure the multi-level 3-D microstructures are clean.

Since the top level can be constructed as the routine photolithography without any trouble on the patterned bottom microstructures, the sealing location and opening locations on the bottom level of microstructures can be designed and formed in the top level of microstructures much flexibly. In addition, since the top layer is constructed from the liquid photo resist, the top layer and the bottom layer can be completely contacted to avoid the micro gas-gap from the coarse surface, leading to intensive crosslinking reaction between the two contacting layers using the standard photolithography procedure. 


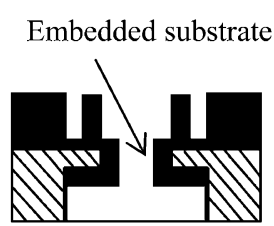

(i)

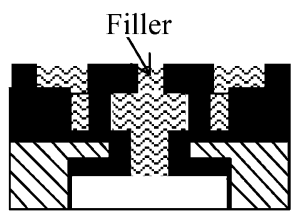

(v)

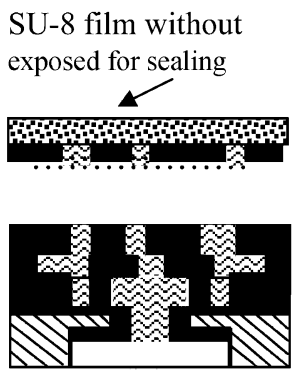

(ix)

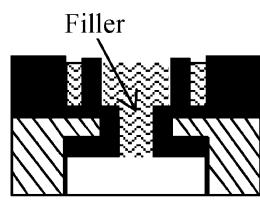

(ii)

SU-8 layer for the $3^{\text {rd }}$ level structure

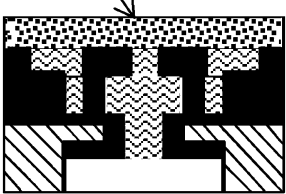

(vi)

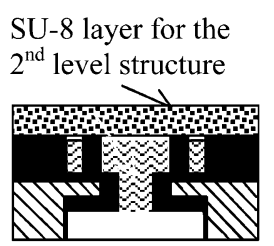

(iii)
The $2^{\text {nd }}$ level

microstructure

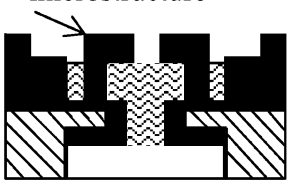

(iv)

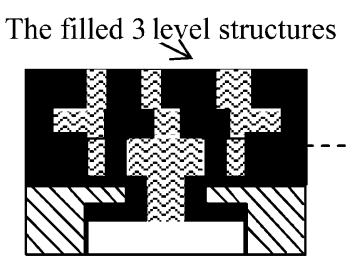

(vii)
The $\mathrm{N}^{\text {th }}$ level microstructure
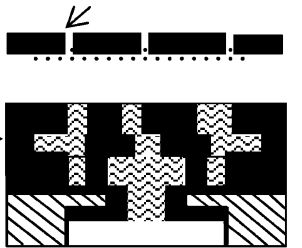

(viii)
Exposed SU-8 film as sealing layer

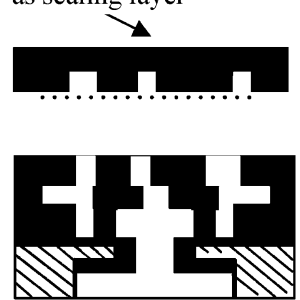

(x)

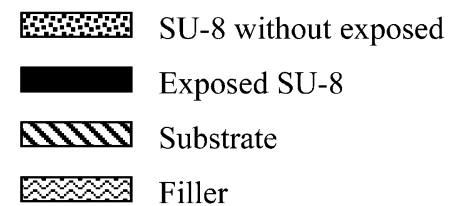

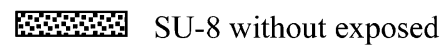

W110 Substrate

$\approx 2$ Filler

FIG. 1. Schematic diagram for the fabrication of multi-level 3-D microstructures by the phase inversion process: (i) Construct the first layer of micro structures with embedded structures on the substrates by the routine UV-LIGA process; (ii) Fill the filler into the $1^{\text {st }}$ layer micro structures; (iii) Spin coating the $2^{\text {nd }}$ SU- 8 layer for the $2^{\text {nd }}$ level microstructure; (iv) Construct the $2^{\text {nd }}$ level SU-8 microstructure by the routine UV-LIGA process; (v) Fill the filler into the 2 level micro structures; (vi) Spin coating the $3^{\text {rd }}$ SU-8 layer for the $3^{\text {rd }}$ level microstructure; (vii) Fabricate the $3^{\text {rd }}$ level SU- 8 micro structure by routine UV-LIGA process and fill the filler in to the $3^{\text {rd }}$ level micro structures; (viii) Fabricate several level microstructures by repeating (ii)-(iv) to form desired levels of microstructures; (ix) Spin coating the last SU-8 layer for sealing; (x) Seal the multi-level microstructures by solidification of the SU-8 layer after exposure under UV-light.

Clearly, the key issue in this process is to find suitable fillers that can reversibly change their phases, from liquid to solid before constructing the top layer, and then from solid to liquid after finishing all construction, either by adjusting operating temperature or by solvents. In addition, there are three basic requirements for this kind of fillers. Firstly, this kind of fillers should have enough low viscosities in liquid phase. Secondly, the melting temperature of the fillers used should be compatible to the common photolithography process of photo resists (e.g. SU-8) [12], or the melting temperature should be around or above the pre-baking and post-baking temperature of the photo resist but lower than the decomposing temperature of the photo-resist, and the fillers should not be dissolved into the developer for the photo resist. For SU-8, the melting temperature range of these fillers can be about $70 \sim 200^{\circ} \mathrm{C}$ according the SU-8 photolithography process and the thermal stability of SU-8 [12]. Thirdly, the fillers can be dissolved by water or some kind of solvent that will not dissolve the cured photo resists. A kind of high melting temperature wax, such as mondang wax $\left(\mathrm{d}=1.09 \mathrm{~g} / \mathrm{cm}^{3}, \mathrm{Tm}=75 \sim 87^{\circ} \mathrm{C}\right.$, Guangzhou Yu Feng Chemical
Trade Co., Ltd), or Polyethylene (PE) wax (XH-200, Tm = $105 \sim 115^{\circ} \mathrm{C}$, XH 206, $\mathrm{Tm}=135 \sim 145{ }^{\circ} \mathrm{C}$, Hero Tianjin International Trade Co., Inc.), or Polyvinyl Alcohol $\left(\mathrm{Mw}=31000 \sim 50000, \mathrm{Tm}=200^{\circ} \mathrm{C} ; \mathrm{d}=1.267 \mathrm{~g} / \mathrm{cm}^{3}\right.$, Aldrich), etc., will be very suitable for these purposes. These waxes can be dissolved by tetrahydrofuran or acetone and the PVA can be dissolved in hot water. While the crosslinking SU-8 photo resists have enough resist to these solvents. Thus, the fillers in liquid phase under a suitable temperature can flow-fill into the microstructures (i. e. channels, mixtures, orifices, chambers) by forming a liquid surface a little bit below the wall of microstructures to prevent from overflowing to other parts when the next layer of liquid SU-8 is coating on. After they change to solid by reducing the temperature, a uniform solid surface together with the patterned structures is formed, which acts as a supporting substrate for the liquid photo resist. Since this process is suitable for liquid coating, the interfaces which may be filled by air bubbles between two layers using other solid thermal bonding process (i.e. hot embossing) can be avoided, particularly for large areas of uneven surfaces. Thereby, a complete contact 
between layers can be obtained that will result in a strong binding.

Using this phase inversion process, many complex multi-layer 3-D microstructures can be constructed although some of them cannot be built up using multi-layer fabrication process mentioned by some reports $[11,21]$. In the following, some typical multi-level 3-D microstructures will be demonstrated using SU-8 as constructing materials and mondang wax or PE wax as fillers.

Figure 2 shows the typical complex 2-level 3-D microchannels with flexible connectors using the phase inversion process. In Fig. 2(a), the transparent optical photograph clearly indicates the first layer channels is covered by the second layer channels, which can have lots of opened connecting parts in the first layer channels to the second layer channels for exchanging information, which may be used as mixers for two-layer liquid-liquid mixing or liquid-gas mixing. Their cross-section optical image (see Fig. 2(b)) suggests that the bottom surface of the second layer channels is connected with the top surface of the first layer channels with many connecting parts. Figure 2(b) shows that the sealing surface is uniformly flat without any blockage and deformation, which can be further evidenced by their Scanning Electron Microscope (SEM) images for some local places (see Fig. 2(c) and 2(d)). Figure 2(c) clearly

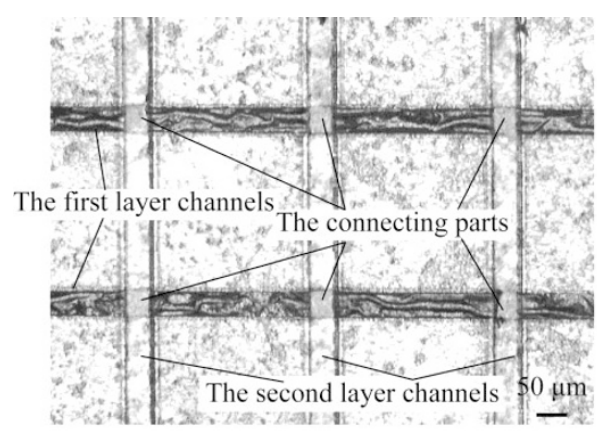

(a)

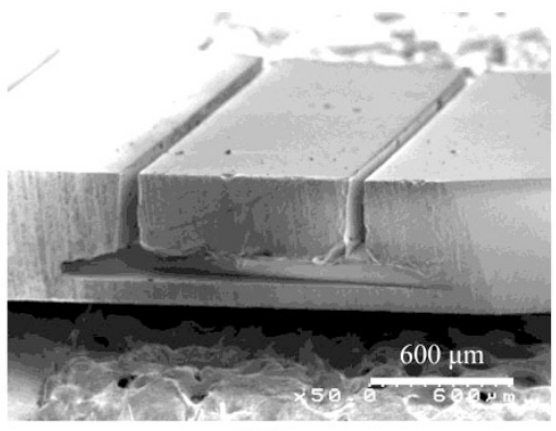

(c) shows that the $80 \mu \mathrm{m}$ wide channels in the top level are opened and connected with the $80 \mu \mathrm{m}$ depth channels in the bottom level at the arrowed locations. Figure 2(d) shows the cross-section images of one bottom $400 \mu \mathrm{m}$ wide channel and one top $80 \mu \mathrm{m}$ wide channel, where the top surface (i) of the bottom channel has the same height as the bottom surface (ii) of the top channel along the dashed line, indicating the depth of the bottom channel about $80 \mu \mathrm{m}$.

Figure 3 shows SEM images for other two typical 2 level 3-D microstructures with channels connected with large orifices or chambers. Figure 3(a) is one large chamber (diameter of 2000 $\mu \mathrm{m})$ with doped bottom U-shaped channel $(80 \mu \mathrm{m})$ inside, suggesting no trace blockage in the channels and the very smooth bottom surface of the chamber. This kind of design may be used for further fabrication of some functional materials inside the channels as sensing units. When the sensing function is finished, the microfluid in the chamber can leave the chamber by directly entering the bottom U-shape channels. Figure 3(b) and 3(c) are the reversed design of Fig. 3(a) with two top channels $(80 \mu \mathrm{m})$ passing through the large chamber (diameter of $1800 \mu \mathrm{m}$ ). Since there are some interface effect between the wax and the wall of the preformed chamber and some volume change of wax during the pre-baking and post-baking of the top layer of SU-8 photo resist, the edge parts in the chamber are not as uniform as those

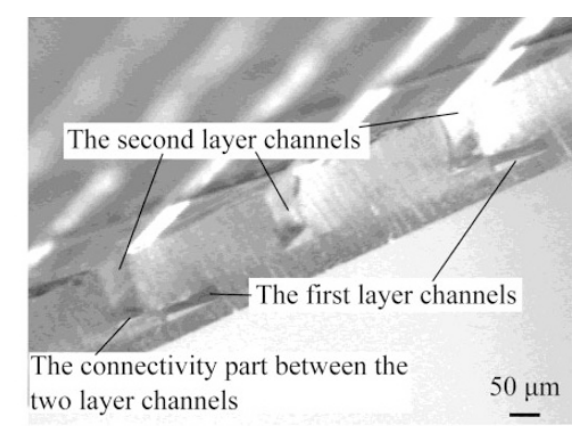

(b)

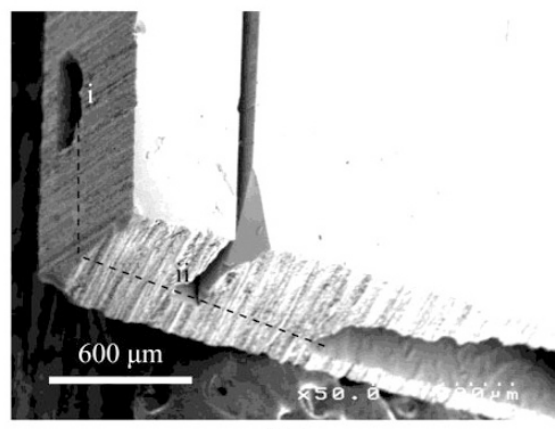

(d)

FIG. 2. Connected two-layer 3-dimensional microchannels. (a) The flat optical image of one cross-connected two-layered 3-D microchannels; (b) The optical images of the cross-section for the connected two-layer 3-D microchannels; (c) The SEM image on the cross-section and two channels at the $2^{\text {nd }}$ layer; (d) The SEM image with two-layered one channel at the bottom layer and one channel at the top layer. 


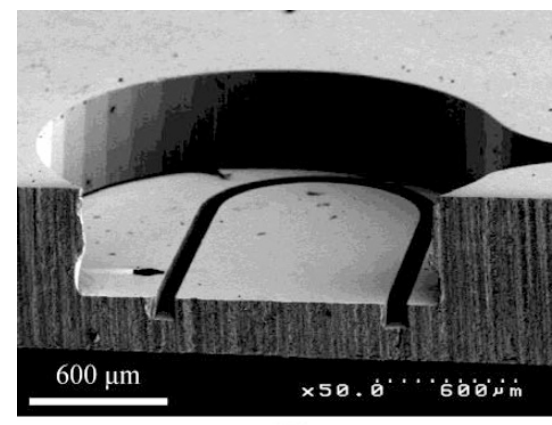

(a)

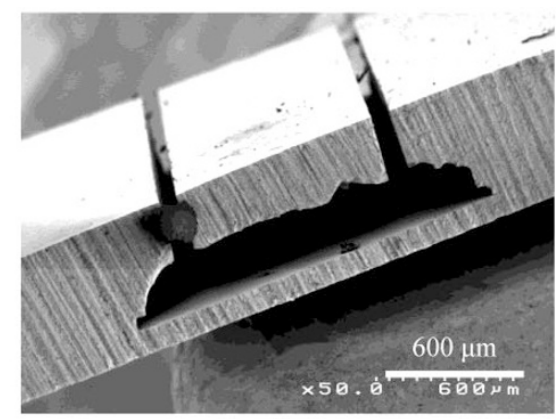

(b)

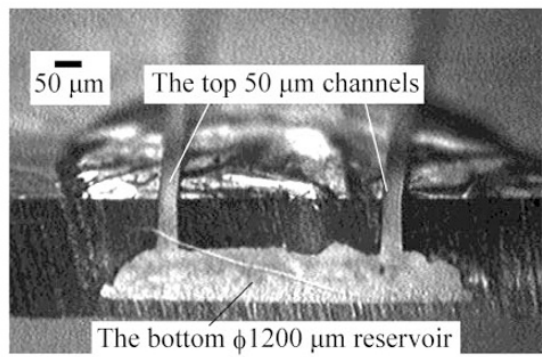

(c)

FIG. 3. Typical examples of microstructures with connecting parts. (a) The SEM image of one big reservoir with one U-shape channels in the bottom; (b) The SEM image of one big reservoir connecting with two top channels; (c) The optical image of the big reservoir connecting with two top channels clearly showing the opening parts of the two channels on the top of the reservoir.

in Fig. 3(a) and show some convex edge-bird effects. But the opening between the chamber and the two channels are very perfect, as shown by the optical microscope image (see Fig. $3(\mathrm{c})$ ).

In the microfabrication, another important field is to sealing very shallow channels with long span, which is also difficult since the blockage occurs often. Using the developed phase inversion process, it will become easy for channels with depth of less than $50 \mu \mathrm{m}$ and span more than $300 \mu \mathrm{m}$. Figure 4 shows the optical image (a) and the SEM image (b) of the cross-section of one channel with $40 \mu \mathrm{m}$ depth and $350 \mu \mathrm{m}$ width, which has sharp surrounding rims and abrupt sidewall-to-bottom transitions without any significant blockage.

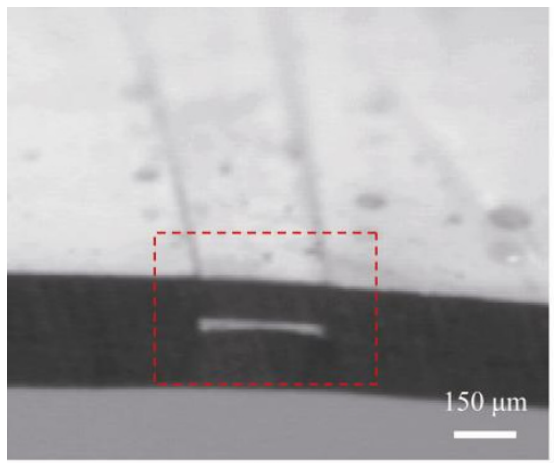

(a) post-backed at $150^{\circ} \mathrm{C}$ for $30 \mathrm{~min}$, and then tested by pumping the tetrahydrofuran solution through the micro-channels at a flow rate of $\sim 15.0 \mathrm{ml} / \mathrm{min}$ and a frequency of $2 \mathrm{~Hz}$ for more than 8 hours, corresponding to a calculated dynamic pressure drop across the channels about 5.3 MPa. The result suggests that no tracing leakage was observed. The sealed microfluidic channels (with width of $60 \mu \mathrm{m}$ and a sealed layer of $400 \mu \mathrm{m}$ thick) were also tested under the static air pressure, indicating that these two layers cannot be debonded even at a pressure of $10 \mathrm{MPa}$ overnight. When the sealed layer was reduced to $60 \mu \mathrm{m}$ thick, the SU-8 film without bonding with the sub-layer is broken while the SU-8 film bonding with the sub-layer is still united together. These results indicate that the sealing process preserves the

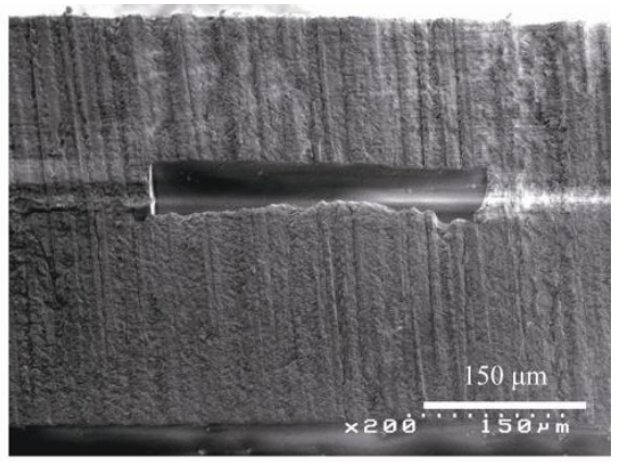

(b)

FIG. 4. One typical sealed shallow microchannel with height of $40 \mu \mathrm{m}$ and width of $350 \mu \mathrm{m}$. (a) The optical image of one shallow microchannel; (b) The SEM image of the cross-section of this shallow microchannel (dashed square in (a)).

Unlike other solid bonding process (e.g. hot-embossing), the interlayer for the sealing between the two layers cannot be envisioned since a uniform and complete contact interface is formed during sealing process using the liquid photo resist (e.g. SU-8) (see Fig. 2, Fig. 3 and Fig. 4), which will lead to ultra-strong bonding between two contacting layers. In order to investigate the bonding strength, samples of the multi-level microchannels with width of $60 \mu \mathrm{m}$, depth of $400 \mu \mathrm{m}$ and length of $25 \mathrm{~cm}$ are sealed by a layer of $400 \mu \mathrm{m}$ thick SU-8 and potential to reach the binding as strong as the bulk photoresist (i.e. SU-8 100) [22].

In summary, one process based on the phase inversion of fillers inside the preformed 3-D microstructures has been successfully developed for multi-level 3-D microstructure construction using SU-8 as model constructing materials. This process makes it possible to seal channels with depth no more than $40 \mu \mathrm{m}$, and to construct desired multi-level 3-D microstructures with connectors at necessary locations flexibly 
with interlayer bonding as robust as the bulk constructing materials (e.g. SU-8).

This work is financially supported by the National Science Foundation of China (No. 50971010), New Teacher Foundation of Beihang University (211109), New Teacher Funds of Education Ministry of China (2008-00061025) and SRF and SEM funds of Education Ministry of China.

Received 31 March 2010; accepted 5 May 2010; published online 18 May 2010.

\section{References}

1. H. A. Stone and S. Kim, AIChE J. 47, 1250(2001). doi:10.1002/aic.690470602.

2. B. Zhao, J. S. Moore and D. J. Beebe, Science 291, 1023(2001). doi:10.1126/science.291.5506.1023.

3. C. Wiles, P. Watts, S. J. Haswell and E. Pombo-Villar Lab Chip 4, 171(2004). doi:10.1039/b400280f.

4. W. Ehrfeld, V. Hessol and H. Lowe, Microreactors: New technology for modern Chemistry, Wiley-VCH, New York, 2000.

5. P. Watts and S. J. Haswell, Drug Discovery Today 8, 586 (2003). doi:10.1016/S1359-6446(03)02732-6.

6. Y. Song, C. S. S. R. Kumar and J. Hormes, Small 4, 698 (2008). doi:10.1002/smll.200701029.

7. Y. Song, L. L. Henry and W. T. Yang, Langmuir 25, 10209 (2009). doi:10.1021/1a9009866.

8. Y. Song and L. L. Henry, Nanoscale Res. Lett. 4, 1130 (2009). doi:10.1007/s11671-009-9369-8.

9. P. Watts and S. J. Haswell, Curr. Opin. Chem. Biol. 7, 380 (2003). doi:10.1016/S1367-5931(03)00050-4.

10. H. Pennemann, P. Watts, S J Haswell, V. Hessel and H. Löwe, Org. Process Res. Dev. 8, 422 (2004). doi:10.10 21/op0341770.
11. M. Castano-Álvarez, M. T., Fernández-Abedul A.Costa-García, M. Agirregabiria, L. J. Fernández, J. M. Ruano-López and B. Barredo-Presa, Talanta 80, 24 (2009). doi:10.1016/j.talanta.2009.05.049.

12. Y. Song, C. S. S. R. Kumar and J. Hormes, J. Micromech. Microeng. 14, 932(2004). doi:10.1088/0960-1317/14/7/ 013.

13. D. Sander, R. Hoffmann, V. Relling and J. Muller, J. Microelectron. Syst. 4, 81 (1995). doi:10.1109/ 84.388116.

14. J Gobet, F. Cardot, J. Bergqvist and F. Rudolf, J. Micromech. Microeng. 3, 123 (1993). doi:10.1088/09601317/3/3/007.

15. O. Lehmann and M. Stuke, Science 270, 1644 (1995). doi:10.1126/science.270.5242.1644.

16. R. J. Jackman, S. T. Brittain, A. Adams, M. G. Prentiss and G. M. Whitesides, Science 280, 2089 (1998). doi:10.1126/science.280.5372.2089.

17. A. A. Ayon, R. A. Braff, R. Bayt, H. H. Sawin and M. A. Schmidt, J. Electrochem. Soc. 146, 2730 (1999).

18. R. J. Jackman, T. M. Floyd, R. Ghodssi, M. A. Schmidt and K. F. Jensen, J. Micromech. Microeng. 11, 263 (2001). doi:10.1088/0960-1317/11/3/316.

19. C. Lin, G. Lee, B. Chang and G. Chang, J. Micromech. Microeng. 12, 590 (2002). doi:10.1088/0960-1317/12/ $\underline{5 / 312}$.

20. H. Lorenz, M. Despont, N. Fahrni, J. Brugger, P. Vettiger and P. Renaud, Sensors and Actuators A: Physical A 64, 33 (1998). doi:10.1016/S0924-4247(98)80055-1.

21. Z. Peng, Z. Ling, M. Tondra, C. Liu, M. Zhang, K. Lian, J. Goettert and J. Hormes, J. Microelect. Syst. 15, 708 (2006).

22. A. McAleavey, G. Coles, R. L. Edwards and W. N. Sharpe, Materials science of microelectromechanical systems (MEMS) devices; Proceedings of the Symposium, Boston, MA, USA, Dec. 1-2, pp. 213-218 (1998). 